\title{
Structural Credit Risk Model with Jumps Based on Uncertainty Theory
}

\section{Hong Huang}

Shandong Youth University of Political Science

Yufu Ning ( $\nabla$ yufuningsd@163.com )

Shandong Youth University of Political Science https://orcid.org/0000-0003-3962-6978

\section{Research Article}

Keywords: structural credit risk model with jumps, default belief degree, uncertainty theory, credit derivatives pricing.

Posted Date: November 5th, 2021

DOl: https://doi.org/10.21203/rs.3.rs-888535/v1

License: (c) (1) This work is licensed under a Creative Commons Attribution 4.0 International License. Read Full License 


\title{
Structural credit risk model with jumps based on uncertainty theory
}

\author{
Hong Huang ${ }^{1,2}, \quad$ Yufu Ning ${ }^{1,2 *}$ \\ ${ }^{1}$ School of Information Engineering, Shandong Youth University of Political Science, jinan \\ 250103, China. \\ ${ }^{2}$ Key Laboratory of Intelligent Information Processing and Information Security in Universities \\ of Shandong, Jinan 250103, China \\ *Corresponding author: yufuningsd@163.com
}

\begin{abstract}
Traditional finance studies of credit risk structured models are based on the assumption that the price of the underlying asset obeys a stochastic differential equation. However, according to behavioral finance, the price of the underlying asset is not entirely stochastic, and the credibility of financial investors also plays a very important role in asset prices. In this paper we introduce uncertainty theory to describe these credibility of investors and propose a new credit risk structured model with jumps based on the assumption that the underlying asset is described by an uncertain differential equation with jumps. The company default belief degree formula, zero coupon bond value and stock value formula are formulated. Company bond credit spread and credit default swap (CDS) pricing are studied as applications of the proposed model in uncertain markets.
\end{abstract}

Keywords. structural credit risk model with jumps, default belief degree, uncertainty theory, credit derivatives pricing.

\section{Introduction}

Credit risk is the risk that a party to a contract will not be able to perform or fulfill its stated obligations during the transaction due to bankruptcy or other serious economic problems. For a long time, credit risk has been considered as one of the main negative factors affecting the development of the financial. After the outbreak of the global financial crisis in 2008, the management and control of credit risk has become the most important topic of quantitative finance research.

The belief degree of default is a measure of credit risk. There are two major schools of thought in the study of credit risk today, one is the structured model and the other is the parsimonious model. In this paper, the structured model is used to study the company credit risk. 
Merton(1974) used the Black-Scholes(1973) formula to create the first credit structured model. This approach has now evolved into a large system of models that occupy an important position in bond pricing and default probability measurement in bond pricing and default belief degree measures. The KMV model(2000), developed by Moody based on a structured model, is now one of the most popular credit risk management models in the financial market. Merton model assumes that the value of a company's asset satisfied geometric Brownian motion. It can only describe the continuous changes in the company's asset value, but ignore the abnormal changes in assets caused by a series of abnormal conditions such as the Brexit vote and the epidemic. Xue and Wang(2008) investigated the structured model of credit risk driven by Lévy process. Based on the theory of stochastic analysis of Lévy process, they analyze the default belief degree, bond value and credit spread of company and obtain their analytical expressions. Assumed that the underlying stock price satisfies a geometric double-exponential jump-diffusion process, and the default occurs when the company stock price falls below the default threshold for the first time, $\mathrm{Wu}$, Tian and Chen(2008) proved a structured model for pricing equity default swaps.

The above studies on structured models of credit risk are conducted in the framework of belief degree theory, which we can use only when the belief degree distribution is close enough to the true frequency. In order to get the actual frequency we need to have sufficient sample data and use statistical methods to infer the belief degree distribution. However, due to market or technical reasons, the problems we face in practice sometimes do not allow us to obtain sample data, many investors usually take the advice of experts as their degree of belief in some financial events and use this as the basis for decision-making. Beliefs play an important role in actual financial practice. In order to portray this belief reasonably, $\operatorname{Liu}(2010,2021)$ proposed uncertainty theory in 2007 and refined into a branch of mathematics based on normality, duality, subadditivity and the product axiom in 2010. In 2009, $\mathrm{Liu}(2009)$ defined the uncertain process and uncertain differential equation, the uncertain differential equation corresponds to the stochastic differential equation in the B-S model. Using this uncertain differential equation to describe stock price, Liu established an uncertain stock price model and gave the pricing formula of standard European options under uncertainty theory. The uncertainty theory was quickly applied to practice, and it also attracted the attention of scholars from all over the world. Chen(2011) proved American option pricing formulas based on the uncertain stock price model above. Peng and Yao(2011) proved a stock model with mean-reverting process and the corresponding option pricing formulas for uncertain 
markets. Sun and $\mathrm{Su}(2017)$ proposed a mean-reverting stock model with floating interest rate in the uncertain financial markets and then employed it to the European option and American option in the uncertain markets. $\mathrm{Wu}$, Zhuang(2018) introduced uncertain theory into credit derivatives, and developed an uncertain formula for credit default swap. Gao, Liu(2021) used uncertain differential equation to dispose of the foreign exchange rate, and investigated an American barrier option of currency model.

To describe a discontinuous uncertain system, Liu(2008) defined an uncertain renewal process in 2008. Yao(2012,2015,2021) established an uncertain calculus on the uncertain renewal process, and proposed a uncertain differential equation with jumps, then gave a sufficient condition for this equation having a unique solution and some stability theorems. $\mathrm{Yu}(2012)$ proposed a stock model with jumps for uncertain financial markets, and derived the pricing formulas for European call and put options with jumps. By means of uncertain differential equation with jumps, Ji and Zhou(2015) proposed a stock model which contains both the positive jumps and the negative jumps and they also proved European option pricing formulas. Based on these European option pricing formulas with jumps

this paper will discuss the measurement of credit risk, where the company's assets are described by uncertain differential equation with jumps.

This paper is organized as follows. In section 2, we introduce some useful concepts of uncertain process and uncertain differential as needed. Then a structural credit risk model with jumps is proposed in section 3. In section 4, as an application of the credit risk structuring model, we discuss the pricing of credit derivatives, including credit spreads and credit default swap. Section 5 is the conclusion of this paper.

\section{Preliminaries}

Uncertainty theory was founded by $\mathrm{Liu}^{[6]}$ to provide a mathematical model for dealing uncertain phenomena in human system. Let $(\Gamma, L, M)$ be an uncertainty space with an uncertain measure $M$, and the uncertain measure is a set function satisfied the normality, self-duality, countable sub-additivity, and product measure axioms. An uncertain variable $\xi$ is a measurable function from an uncertainty space to the set of real numbers.

Definition $\mathbf{1}^{[6]}$. The uncertainty distribution $\Phi: \mathfrak{R} \rightarrow[0,1]$ of an uncertain variable $\xi$ is defined by 


$$
\Phi(x)=M\{\xi \leq x\} .
$$

Definition $2^{[6]}$. Let $\xi$ be an uncertain variable. Then the expected value of $\xi$ is defined by

$$
E[\xi]=\int_{0}^{+\infty} M\{\xi \geq r\} d r-\int_{-\infty}^{0} M\{\xi \leq r\} d r .
$$

And an uncertain process is a sequence of uncertain variables indexed by time and space, In this paper we will use the most important two uncertain processes: canonical Liu process and renewal process.

Definition $3^{[6]}$. An uncertain process $C_{t}$ is called a canonical Liu process if

(i) $C_{0}=0$ and almost all sample paths are Lipschitz continuous;

(ii) $C_{t}$ has stationary and independent increments;

(iii) $C_{t+s}-C_{t}$ is a normal uncertain variable with expected value 0 and variance $t^{2}$,and the following uncertainty distribution

$$
\Psi(x)=\left(1+\exp \left(-\frac{\pi x}{\sqrt{3} t}\right)\right)^{-1} .
$$

Definition $4^{[6]}$. An uncertain process $N_{t}$ is called a renewal process if

$$
N_{t}=\max _{n \geq 0}\left\{n \mid S_{n} \leq t\right\},
$$

where $\xi_{1}, \xi_{2} \cdots$ be independent identical distribution positive uncertain variables, $S_{n}=\xi_{1}+\xi_{2} \cdots+\xi_{n}$ for $n>1$, and $S_{0}=0$.

Definition $5^{[7]}$. Let $X_{t}$ be an uncertain process and $C_{t}$ be a canonical Liu process. For any partition of closed interval $[a, b]$ with $a=t_{1}<t_{2} \cdots<t_{k+1}=b$, the mesh is defined as

$$
\Delta=\max _{1 \leq i \leq k}\left|t_{i+1}-t_{i}\right| \text {. }
$$

Then the uncertain integral of $X_{t}$ with respect to $C_{t}$ is

$$
\int_{a}^{b} X_{t} d C_{t}=\lim _{\Delta \rightarrow 0} \sum_{i=1}^{k} X_{t} \cdot\left(C_{t_{i+1}}-C_{t_{i}}\right)
$$

Definition ${ }^{{ }^{[14]}}$. Let $X_{t}$ be an uncertain process and $N_{t}$ be a renewal process. For any partition of closed interval $[a, b]$ with $a=t_{1}<t_{2} \cdots<t_{k+1}=b$, the mesh is defined as

$$
\Delta=\max _{1 \leq i \leq k}\left|t_{i+1}-t_{i}\right| .
$$

Then the uncertain integral of $X_{t}$ with respect to $N_{t}$ is

$$
\int_{a}^{b} X_{t} d N_{t}=\lim _{\Delta \rightarrow 0} \sum_{i=1}^{k} X_{t} \cdot\left(N_{t_{i+1}}-N_{t_{i}}\right)
$$


Next, we introduce uncertain differential with respect to both $C_{t}$ and $N_{t}$.

Definition $7^{[16]}$. Let $C_{t}$ be a canonical Liu process, $N_{t}$ a renewal process and $Z_{t}$ an uncertain process, if there exist uncertain processes $\mu_{s}, \sigma_{s}, \eta_{s}$ such that

$$
Z_{t}=Z_{0}+\int_{0}^{t} \mu_{s} d s+\int_{0}^{t} \sigma_{s} d C_{s}+\int_{0}^{t} \eta_{s} d N_{s},
$$

for any $t \geq 0$, then $Z_{t}$ is said to have an uncertain differential

$$
d Z_{t}=\mu_{t} d t+\sigma_{t} d C_{t}+\eta_{t} d N_{t} .
$$

And if $f, g$ and $h$ are some given functions, then

$$
d X_{t}=f\left(t, X_{t}\right) d t+g\left(t, X_{t}\right) d C_{t}+h\left(t, X_{t}\right) d N_{t}
$$

is called an uncertain differential equation with jumps.

For example let $\alpha, \beta$ and $\gamma$ be real numbers, we will use the following uncertain differential equation with jumps in the rest of this paper

$$
d X_{t}=\alpha X_{t} d t+\beta X_{t} d C_{t}+\gamma X_{t} d N_{t}
$$

And it is easy to verify that this equation has a solution

$$
X_{t}=X_{0}(1+\gamma)^{N_{t}} \exp \left\{\alpha t+\beta C_{t}\right\}
$$

Lemma $1^{[6]}$. Let $f(x)$ be a Riemann integrable function, then for any $a>0$, Liu integral $Y=\int_{0}^{a} f(t) d C_{t}$ is a is a normal uncertain variable, and

$$
Y \sim N\left(0, \int_{0}^{a}|f(t)| d t\right)
$$

Lemma $1^{[6]}$. Let $N_{t}$ be a renewal process with iid positive uncertain inter-arrival times $\xi_{1}, \xi_{2}, \xi_{3} \cdots$ with distribution function $\Phi(x)$ and $S_{n}=\xi_{1}+\xi_{2}+\cdots+\xi_{n}$, then $N_{t}$ has an uncertainty distribution

$$
\Upsilon_{t}(x)=M\left\{N_{t} \leq[x]\right\}=M\left\{S_{[x]+1} \leq t\right\}=1-\Phi\left(\frac{t}{[x]+1}\right)
$$

where $[x]$ represent the maximal integer less than or equal to $x$.

\section{Structural Credit Risk Model with jumps}

\subsection{Default belief degree and Default Distance}

Let $p_{t}$ be the bond price, and $V_{t}$ the company value price. Assume that the company value price follows a geometric canonical process with jumps. Then we propose an uncertain company value model as follows 


$$
\left\{\begin{array}{l}
d p_{t}=r p_{t} d t \\
d V_{t}=\mu V_{t} d t+\sigma V_{t} d C_{t}+\eta V_{t} d N_{t},
\end{array} \quad t \in[0, T],\right.
$$

here $r$ is the risk-less interest rate, $\mu, \sigma$ and $\eta>0$ is the company value process drift, diffusion and jump coefficients. $C_{t}$ is a standard canonical Liu process, $N_{t}$ is a renewal process with positive uncertain inter-arrival times $\xi_{1}, \xi_{2}, \xi_{3} \cdots$. Assume that $C_{t}$ and $N_{t}$ are independent process, $\xi_{1}, \xi_{2}, \xi_{3} \cdots$ are $i i d$ with distribution function $\Phi(x)$.

It is easy to verify the company value process in uncertain market $V_{t}$ is

$$
V_{t}=V_{0}(1+\eta)^{N_{t}} \exp \left(\mu t+\sigma C_{t}\right), t \in[0, T] .
$$

Structured credit risk model was founded by Merton in 1974 which was pricing models based on Black-Scholes option pricing theory. In this model, the credit relationship was treated as an option transaction, the company assets $V_{t}$ was held by creditors and shareholders together, consisted of zero coupon discount bonds and stocks. Assume that the market is friction-less, there are no government taxes, trading commissions, or bankruptcy reorganization costs, the dividend distribution policy does not affect the value movement of the company, and that the company's default time can only be the bond maturity date $T$. $K$ is the par value of company bonds. The profits of company creditors and company shareholders at time $t$ are: if $V_{T} \leq K$, the company will default, the creditor gets $V_{T}$ and the shareholder gets 0 , If $V_{T}>K$, the company will not default, the creditor gets $K$ and the shareholder gets $V_{T}-K$.

Theorem 1. Assume that $N_{t}$ is an uncertain renewal process with independent identical distribution uncertain inter-arrivals $\xi_{1}, \xi_{2}, \xi_{3} \cdots$ whose uncertainty distribution is $\Phi(\cdot)$. If the company asset is described as (3), and assume that the company's default time can only be $T$, then the company default belief degree $p$ and default distance $d$ in uncertain market are

$$
p=\sup _{n \geq 0} \mathrm{~N}(d) \wedge\left(1-\Phi\left(\frac{T}{n+1}\right)\right) .
$$

and

$$
d=\frac{\ln \left(\frac{K}{V_{0}}\right)-\mu T-n \ln (1+\eta)}{\sigma T} .
$$

where $\mathrm{N}(\cdot)$ is a standard normal uncertainty distribution 


$$
\mathrm{N}(x)=\left(1+\exp \left(-\frac{\pi x}{\sqrt{3}}\right)\right)^{-1}
$$

Proof. According to the definition of company default belief degree and company value process (3), we can get

$$
\begin{aligned}
M\left\{V_{T}<K\right\} & =M\left\{V_{0}(1+\eta)^{N_{T}} \exp \left(\mu t+\sigma C_{T}\right)<K\right\} \\
& =M\left\{\mu T+\sigma C_{T}+N_{T} \ln (1+\eta)<\ln \left(\frac{K}{V_{0}}\right)\right\} \\
& =M\left\{\sigma C_{T}+N_{T} \ln (1+\eta)<\ln \left(\frac{K}{V_{0}}\right)-\mu T\right\} \\
& =M\left\{\bigcup_{n=0}^{\infty}\left(\sigma C_{T}<\ln \left(\frac{K}{V_{0}}\right)-\mu T-n \ln (1+\eta)\right) \cap\left(N_{T}=n\right)\right\} \\
& =\sup _{n \geq 0} M\left\{C_{T}<\frac{\ln \left(\frac{K}{V_{0}}\right)-\mu T-n \ln (1+\eta)}{\sigma}\right\} \wedge M\left(N_{T} \leq n\right),
\end{aligned}
$$

Since $C_{T}$ a normal uncertain variable with expected value 0 and variance $T^{2}$, let $\mathrm{N}(\cdot)$ be a standard normal uncertainty distribution and

$$
d=\frac{\ln \left(\frac{K}{V_{0}}\right)-\mu T-n \ln (1+\eta)}{\sigma T}
$$

which is called default distance of company and then get the following company default belief degree

$$
p=M\left\{V_{T}<K\right\}=\sup _{n \geq 0} \mathrm{~N}(d) \wedge\left(1-\Phi\left(\frac{T}{n+1}\right)\right) .
$$

The prove is completed.

\subsection{Risk-neutral uncertainty measure}

Let $E\left(t, V_{t}, T\right)$ and $D\left(t, V_{t}, T\right)$ be the value of the stock and zero coupon bonds issued by the company at time $t$.As previously discussed, if $V_{T}<K$, company bond value $D\left(0, V_{0}, T\right)$ will be $V_{T}$, and stock value $E\left(0, V_{0}, T\right)$ will be 0 . Contrarily, if $V_{T}>K$, the company zero coupon bond value $D\left(0, V_{0}, T\right)$ will be $K$ and stock value $E\left(0, V_{0}, T\right)$ will be $V_{T}-K$. That is,

$$
E\left(0, V_{0}, T\right)=\max \left\{V_{T}-K, 0\right\}=\left\{\begin{array}{c}
V_{T}-K, \text { if } V_{T} \geq K, \\
0, \quad \text { if } V_{T}<K,
\end{array}\right.
$$




$$
D\left(0, V_{0}, T\right)=K-\max \left\{K-V_{T}, 0\right\}= \begin{cases}V_{T}, & \text { if } V_{T}<K, \\ K, & \text { if } V_{T} \geq K\end{cases}
$$

It means that stock value is a call option of the company asset and the company zero coupon bond value is a non-default bond minus the value of a put option with company asset as the subject matter. Option pricing requires financial markets to be free of arbitrage opportunities, i.e. "fair prices". According to the no-arbitrage theorem proposed by Yao(2015), the sufficient and necessary condition for uncertain stock model (3) to be no-arbitrage is that the company value process drift coefficient $\mu$ is equal to the risk-free rate $r$.That is, we need a risk-neutral uncertainty measure $M^{Q}$ which satisfies

$$
E^{Q}\left(e^{-r T} V_{T}\right)=V_{0} .
$$

Let $\lambda$ be the expected rate of return on $V_{t}$, then the following equation holds

$$
E\left(e^{-\lambda T} V_{T}\right)=V_{0},
$$

Combining equations (10) and (11), we can derive the following theorem for expectation of $V_{t}$ under the risk-neutral measure.

Theorem 2. Assume company asset value process is described by (3), then expected rate of return on company asset $\lambda$ is

$$
\lambda=-\frac{\ln V_{0}}{T} \ln \left(\int_{0}^{+\infty}\left(\inf _{n \geq 0} \mathrm{~N}\left(-d_{1}\right) \vee \Phi\left(\frac{T}{n+1}\right)\right) d x\right),
$$

and the expectation of $V_{t}$ under the risk-neutral measure is

$$
E^{Q}\left(V_{T}\right)=e^{(r-\lambda) T} \int_{0}^{+\infty}\left(\inf _{n \geq 0} \mathrm{~N}\left(-d_{1}\right) \vee \Phi\left(\frac{T}{n+1}\right)\right) d x,
$$

where $\mathrm{N}(\cdot)$ is a standard normal uncertainty distribution (7), and

$$
d_{1}=\frac{\ln \left(\frac{x}{V_{0}}\right)-\mu T-n \ln (1+\eta)}{\sigma T} .
$$

Proof. We calculate $E\left(V_{T}\right)$ firstly, 


$$
\begin{gathered}
E\left(V_{T}\right)=E\left(V_{0}(1+\eta)^{N_{T}} \exp \left(\mu T+\sigma C_{T}\right)\right) \\
=\int_{0}^{+\infty} M\left((1+\eta)^{N_{T}} \exp \left(\mu T+\sigma C_{T}\right) \geq \frac{x}{V_{0}}\right) d x \\
=\int_{0}^{+\infty} 1-M\left(C_{T} \leq \frac{\ln \frac{x}{V_{0}}-\mu T-N_{T}(1+\eta)}{\sigma}\right) d x \\
=\int_{0}^{+\infty} 1-M\left(\bigcup _ { n = 0 } ^ { + \infty } \left(\left\{\begin{array}{l}
\left.\ln \frac{x}{V_{0}}-\mu T-n \ln (1+\eta)\right) \\
\left.C_{T} \leq \frac{\sigma}{\sigma}\left(N_{T} \leq n\right\}\right)
\end{array}\right) d x\right.\right. \\
=\int_{0}^{+\infty}\left\{1-\left(\sup _{n \geq 0} M\left\{C_{T} \leq \frac{\ln \left(\frac{x}{V_{0}}\right)-\mu T-n \ln (1+\eta)}{\sigma}\right\} \wedge M\left(N_{T} \leq n\right)\right)\right\} d x,
\end{gathered}
$$

Since $C_{T} \sim \mathrm{N}(0, T)$, and let

$$
d_{1}=\frac{\ln \left(\frac{x}{V_{0}}\right)-\mu T-n \ln (1+\eta)}{\sigma T}
$$

then

$$
\begin{aligned}
E\left(V_{T}\right) & =\int_{0}^{+\infty} 1-\left(\sup _{n \geq 0} \mathrm{~N}\left(d_{1}\right) \wedge\left(1-\Phi\left(\frac{T}{n+1}\right)\right)\right) d x \\
& =\int_{0}^{+\infty}\left(\inf _{n \geq 0} \mathrm{~N}\left(-d_{1}\right) \vee \Phi\left(\frac{T}{n+1}\right)\right) d x .
\end{aligned}
$$

Next we prove equation (12) holds. From Equation (11), it follows that

$$
\mathrm{e}^{\lambda T}=\frac{E\left(V_{T}\right)}{V_{0}}=\frac{1}{V_{0}} \int_{0}^{+\infty}\left(\inf _{n \geq 0} \mathrm{~N}\left(-d_{1}\right) \vee \Phi\left(\frac{T}{n+1}\right)\right) d x .
$$

then we can easily get the expected rate of return of $V_{t}$

$$
\lambda=-\frac{\ln V_{0}}{T} \ln \left(\int_{0}^{+\infty}\left(\inf _{n \geq 0} \mathrm{~N}\left(-d_{1}\right) \vee \Phi\left(\frac{T}{n+1}\right)\right) d x\right)
$$

Combining equations (10) and (11), we get 


$$
\begin{aligned}
E^{Q}\left(V_{T}\right) & =e^{(r-\lambda) T} E\left(V_{T}\right) \\
& =e^{(r-\lambda) T} \int_{0}^{+\infty}\left(\inf _{n \geq 0} \mathrm{~N}\left(-d_{1}\right) \vee \Phi\left(\frac{T}{n+1}\right)\right) d x .
\end{aligned}
$$

The prove is completed.

\subsection{Company Stock Value and Zero Coupon Bond Value}

According to Black-Scholes formula, we get the following theorem 3 and 4 about the value of company stock and company zero coupon bond .

Theorem 3. Assume company asset value process is described by (3), the value of company stock at time 0 is

$$
E\left(0, V_{0}, T\right)=\mathrm{e}^{-\lambda T} \int_{K e^{(\lambda-r) T}}^{+\infty}\left(\inf _{n \geq 0} \mathrm{~N}\left(-d_{1}\right) \vee \Phi\left(\frac{T}{n+1}\right)\right) d x
$$

where $\lambda$ is the expected rate of $V_{t}$ with expression(12), $\mathrm{N}(\cdot)$ is a standard normal uncertainty distribution (7) and $d_{1}$ is defined as(14).

Proof. According to Black-Scholes formula ${ }^{[2]}$ of call option

$$
\begin{aligned}
E\left(0, V_{0}, T\right) & =E^{Q}\left[\mathrm{e}^{-r T}\left(V_{T}-K\right)^{+}\right] \\
& =E\left[\left(\mathrm{e}^{-\lambda T} V_{T}-\mathrm{e}^{-r T} K\right)^{+}\right] \\
& =\int_{0}^{+\infty} M\left(\mathrm{e}^{-\lambda T} V_{T}-\mathrm{e}^{-r T} K \geq \theta\right) d \theta \\
& =\int_{0}^{+\infty} M\left(V_{T} \geq \mathrm{e}^{(\lambda-r) T} K+\mathrm{e}^{\lambda T} \theta\right) d \theta,
\end{aligned}
$$

let $x=e^{\lambda T} \theta+e^{(\lambda-r) T} K$, we have

$$
\begin{aligned}
& E\left(0, V_{0}, T\right)=\mathrm{e}^{-\lambda T} \int_{K e^{(\lambda-r) T}}^{+\infty} M\left\{V_{T} \geq x\right\} d x \\
& =\mathrm{e}^{-\lambda T} \int_{K e^{(\lambda-r) T}}^{+\infty} 1-M\left\{V_{T} \leq x\right\} d x \\
& =\mathrm{e}^{-\lambda T} \int_{K e^{(\lambda-r) T}+\infty}^{+\infty} 1-M\left\{\mu T+\sigma C_{T}+N_{T} \ln (1+\eta)<\ln \left(\frac{x}{V_{0}}\right)\right\} d x \\
& =\mathrm{e}^{-\lambda T} \int_{K e^{(\lambda-r) T}}^{+\infty}\left\{1-\left(M\left\{\bigcup_{n=0}^{\infty} C_{T} \leq \frac{\ln \left(\frac{x}{V_{0}}\right)-\mu T-n \ln (1+\eta)}{\sigma}\right\} \cap\left(N_{T}=n\right)\right)\right\} d x
\end{aligned}
$$




$$
=\mathrm{e}^{-\lambda T} \int_{K e^{(\lambda-r) T}}^{+\infty}\left\{1-\left(\sup _{n \geq 0} M\left\{C_{T} \leq \frac{\ln \left(\frac{x}{V_{0}}\right)-\mu T-n \ln (1+\eta)}{\sigma}\right\} \wedge M\left(N_{T} \leq n\right)\right)\right\} d x,
$$

Since $C_{T} \sim \mathrm{N}(0, T)$,

$$
\begin{aligned}
E\left(0, V_{0}, T\right) & =\mathrm{e}^{-\lambda T} \int_{K e^{(\lambda-r) T}}^{+\infty} 1-\left(\sup _{n \geq 0} \mathrm{~N}\left(d_{1}\right) \wedge\left(1-\Phi\left(\frac{T}{n+1}\right)\right)\right) d x \\
& =\mathrm{e}^{-\lambda T} \int_{K e^{(\lambda-r) T}}^{+\infty}\left(\inf _{n \geq 0} \mathrm{~N}\left(-d_{1}\right) \vee \Phi\left(\frac{T}{n+1}\right)\right) d x
\end{aligned}
$$

here $d_{1}$ is defined as $(14)$, and $\mathrm{N}(\cdot)$ is a standard normal uncertainty distribution (7).

The prove is completed.

Similarly, we can obtain the following formula for company zero coupon bond.

Theorem 4. Assume company asset value process is described by (3), the value of company zero coupon bond at time 0 is

$$
D\left(0, V_{0}, T\right)=K \mathrm{e}^{-r T}-\mathrm{e}^{-\lambda T} \int_{0}^{K e^{(\lambda-r) T}}\left(\sup _{n \geq 0} \mathrm{~N}\left(d_{1}\right) \wedge\left(1-\Phi\left(\frac{T}{n+1}\right)\right)\right) d x .
$$

where $\mathrm{N}(\cdot)$ is a standard normal uncertainty distribution, $d_{1}$ is defined as $(14)$ and $\lambda$ is the expected rate of return on $V_{t}$ defined as(12).

Proof. According to Black-Scholes formula ${ }^{[2]}$ of put option

$$
\begin{aligned}
D\left(0, V_{0}, T\right) & =K \mathrm{e}^{-r T}-E^{Q}\left[\mathrm{e}^{-r T}\left(K-V_{T}\right)^{+}\right] \\
& =K \mathrm{e}^{-r T}-E\left[\left(\mathrm{e}^{-r T} K-\mathrm{e}^{-\lambda T} V_{T}\right)^{+}\right] \\
& =K \mathrm{e}^{-r T}-\int_{0}^{+\infty} M\left\{\mathrm{e}^{-r T} K-\mathrm{e}^{-\lambda T} V_{T} \geq \theta\right\} d \theta \\
& =K \mathrm{e}^{-r T}-\int_{0}^{+\infty} M\left\{V_{T} \leq \mathrm{e}^{(\lambda-r) T} K-\mathrm{e}^{\lambda T} \theta\right\} d \theta,
\end{aligned}
$$

let $x=e^{(\lambda-r) T} K-e^{\lambda T} \theta$, we have

$$
\begin{aligned}
D\left(0, V_{0}, T\right) & =K \mathrm{e}^{-r T}-\mathrm{e}^{-\lambda T} \int_{0}^{K e^{(\lambda-r) T}} M\left\{V_{T} \leq x\right\} d x \\
& =K \mathrm{e}^{-r T}-\mathrm{e}^{-\lambda T} \int_{0}^{K e^{(\lambda-r) T}} M\left\{\sigma C_{T}+N_{T} \ln (1+\eta) \leq \ln \frac{x}{V_{0}}-\mu T\right\} d x
\end{aligned}
$$




$$
\begin{aligned}
& =K \mathrm{e}^{-r T}-\mathrm{e}^{-\lambda T} \int_{0}^{K e^{(\lambda-r) T}} M\left\{\bigcup_{n=0}^{\infty} C_{T} \leq \frac{\ln \frac{x}{V_{0}}-\mu T-n \ln (1+\eta)}{\sigma} \cap\left(N_{T}=n\right)\right\} d x \\
& =K \mathrm{e}^{-r T}-\mathrm{e}^{-\lambda T} \int_{0}^{K e^{(\lambda-r) T}}\left(\sup _{n \geq 0} M\left\{C_{T} \leq \frac{\ln \frac{x}{V_{0}}-\mu T-n \ln (1+\eta)}{\sigma}\right\} \wedge M\left(N_{T} \leq n\right)\right] d x \\
& =K \mathrm{e}^{-r T}-\mathrm{e}^{-\lambda T} \int_{0}^{K e^{(\lambda-r) T}}\left(\sup _{n \geq 0} \mathrm{~N}\left(d_{1}\right) \wedge\left(1-\Phi\left(\frac{T}{n+1}\right)\right)\right) d x,
\end{aligned}
$$

here $d_{1}$ is defined as $(17)$, and $\mathrm{N}(\cdot)$ is a standard normal uncertainty distribution (7).

The prove is completed.

\section{Credit Derivatives Pricing}

\subsection{Company Bond Credit Spread}

An important parameter for defaulted bonds is the credit spread. The default spread is the difference between the yield on a defaulted bond and the corresponding equivalent yield on a non-defaulted bond,which compensates investors for the risk taken. In the bond market, credit spread is used as a proxy for the credit risk of corporate bonds. The credit risk of company bonds is inferred by observing the change of credit spread. The higher the credit spread, the higher the risk of the company bond, and the higher the return.

Denote by $C S(t, T)$ the credit spread at moment $t$ for a defaulted bond with maturity $T$, let $K=1$ in $D\left(0, V_{0}, T\right)$ then we can prove the following pricing formula for company bond credit spread in uncertain markets.

Theorem 5. Assume company asset value process is described by (3), the value of company bond credit spread at time 0 is

$$
C S(0, T)=-\frac{1}{T} \ln \left(1-\mathrm{e}^{(r-\lambda) T} \int_{0}^{e^{(\lambda-r) T}}\left(\sup _{n \geq 0} \mathrm{~N}\left(d_{1}\right) \wedge\left(1-\Phi\left(\frac{t}{n+1}\right)\right)\right) d x\right) .
$$

$\lambda$ is the expected rate of return on $V_{t}$ defined as(12), $\mathrm{N}(\cdot)$ is a standard normal uncertainty distribution, $d_{1}$ is defined as(14).

Proof. From the credit spread calculation formula, we get 


$$
\begin{aligned}
C S(0, T) & =-\frac{\ln \left(D\left(0, V_{0}, T\right)\right)-\ln \left(e^{-r T}\right)}{T} \\
& =-\frac{\ln \left(e^{r T} D\left(0, V_{0}, T\right)\right)}{T}
\end{aligned}
$$

here $K=1$ in $D\left(0, V_{0}, T\right)$.

$$
C S(0, T)=-\frac{1}{T} \ln \left(1-\mathrm{e}^{(r-\lambda) T} \int_{0}^{e^{(\lambda-r) T}}\left(\sup _{n \geq 0} \mathrm{~N}\left(d_{1}\right) \wedge\left(1-\Phi\left(\frac{t}{n+1}\right)\right)\right) d x\right) .
$$

The prove is completed.

\subsection{Credit Default Swap (CDS) Pricing}

Credit default swap is the most basic product in the credit derivatives market, firstly issued in the U.S. in 2004 and officially issued in China in September 2016. Credit default swaps provide a certain protection for the purchaser of the contract to avoid the risks of a specific company. The buyer of a contract pays a periodic fee to the seller during the term of the CDS contract, which continues until the end of the contract or an event of default. And the CDS seller will protect the buyer's credit risk for the duration of the contract and will accept the corresponding contract fees. The purchaser of CDS can enforce the right to sell a specified bond issued by the company to the seller at face value when a specific company defaults. In the following, we will discuss the CDS pricing of company bonds based on the credit risk structure model developed above.

Consider a CDS contract with a maturity date $T$, buyer's payment time is $\left(t_{1}, t_{2} \cdots, t_{n}\right)$, the prices of CDS is $\omega$ which is the number we will calculate, and $\delta_{i}=t_{i}-t_{i-1}$ is the time interval between adjacent payment periods. The face value of the company's zero coupon bond is still denoted by $K$. Assume the company can only default at the time $T$, that is the CDS buyer will pay the full premium to the seller, so the total expected cash inflow of the CDS seller is $K \sum_{i=1}^{n} \omega \delta_{i}$, the present value after discounting is

$$
K \omega \sum_{i=1}^{n} e^{-r t_{i}} \delta_{i}
$$

On the other hand, the expected cash outflow of the CDS seller is a contingent liability which contingent on the default or non-default of the company, that is

$$
K-\min \left(K, V_{T}\right)=\max \left(0, K-V_{T}\right),
$$

and the present value after discounting is 


$$
E^{Q}\left[e^{-r T}\left(K-V_{T}\right)^{+}\right]
$$

this can obviously be regarded as a European put option with the underlying company value and the strike price of $K$. According to theorem 4, the present value after discounting is

$$
E^{Q}\left[e^{-r T}\left(K-V_{T}\right)^{+}\right]=\mathrm{e}^{-\lambda T} \int_{0}^{K e^{(\lambda-r) T}}\left(\sup _{n \geq 0} \mathrm{~N}\left(d_{1}\right) \wedge\left(1-\Phi\left(\frac{T}{n+1}\right)\right)\right) d x
$$

Since there is no arbitrage opportunity in the financial market, for the CDS seller, his expected cash inflow and outflow should be equal

$$
K \omega \sum_{i=1}^{n} e^{-r t_{i}} \delta_{i}=e^{-r T} E^{Q}\left[e^{-r T}\left(K-V_{T}\right)^{+}\right]
$$

then we get the CDS price $\omega$

$$
\omega=\frac{e^{-(r+\lambda) T}}{K \sum_{i=1}^{n} e^{-r t_{i}} \delta_{i}} \int_{0}^{K e^{(\lambda-r) T}}\left(\sup _{n \geq 0} \mathrm{~N}\left(d_{1}\right) \wedge\left(1-\Phi\left(\frac{t}{n+1}\right)\right)\right) d x
$$

\section{Conclusion}

Based on uncertainty theory, this paper investigates the problem of credit risk measurement when the company's asset is assumed to be described by an uncertain differential equation with jumps. The formulas for company's default belief degree, zero coupon bond and stock value are obtained. Then these presented results are applied to the pricing research of credit derivatives, and get the pricing formulas of company bond credit spread and credit default swap. This research remedies the shortcomings of traditional credit risk studies that rely solely on stochastic theory for pricing, and has some reference value for practical investment analysis. Further research may consider applying the presented model to actual credit risk measurement, such as deriving the corresponding KMV model and conducting empirical research. Besides, how to measure credit risk when a company's asset is driven by a combination of stochastic and uncertain processes is also an interesting and challenging problem.

\section{Acknowledgements}

This work was supported by Shandong NSF (No. ZR2019BG015), Shandong Provincial Higher Education Science and Technology Plan Project (No. J18KA236).

\section{Declarations}


Conflicts of interest/Competing interests: The authors declare that they have no conflicts of interest.

Availability of data and material: Not applicable.

Code availability: Not applicable.

\section{References}

Black F, Scholes M(1973) The pricing of options and corporate liabilities. Journal of political economy, 3: 637-654.

Crouhy M, Galai D(2000) Mark R. A comparative analysis of current credit risk models. Journal of finance 24: 59-117.

Chen X(2011) American option pricing formula for uncertain financial market. European journal of operational research 8: 32-37.

Gao R, Liu K, Li Z, Lang L(2021) American barrier option pricing formulas for currency model in uncertain environment. Journal of system science and complexity. Online Doi:10.1007/s 11424-021- 0039-y.

Ji X, Zhou J(2015) Option pricing for an uncertain stock model with jumps. Soft computing. 19:3323-3329.

Liu B(2008) Fuzzy process, hybrid process and uncertain process. Journal of uncertain systems 2: 3-16.

Liu B(2009) Some research problem in uncertainty theory, Journal of uncertain systems 2: 3-10.

Liu B(2010) Uncertainty theory: A branch of mathematics for modeling human uncertainty. Berlin: Spring-Verlag.

Liu B(2021) Uncertainty theory, 5th edition, Uncertainty theory laboratory.

Peng J(2011) Yao K.. A new option pricing model for stocks in uncertainty markets. International journal of operations research 8:18-26.

Robert C(1974) On the pricing of corporate debt: the risk structure of interest rates. Journal of finance 29: 449-470.

Sun $Y$, Su T(2017) Mean-reverting stock model with floating interest rate in uncertain environment. Fuzzy optimization and decision making 16: 235-255.

Wu Z, Tian M, Chen Q(2008) Study of pricing model of equity deafult swap. Journal of systems engineering 23: 632-635.

Wu L, Zhuang Y(2018) A new credit derivatives pricing model under uncertainty process. Systems science and control engineering 6: 477-481. 
Xue H, Wang N(2008) Structural credit risk model driven by Lévy process. Journal of shanxi university(Nat. Sci. Ed.) 31: 406-409.

Yao K(2012) Uncertain calculus with renewal process. Fuzzy optimization and decision making 11: 285-297.

Yao K(2015) Uncertain differential equation with jumps. Soft computing 19: 2063-2069.

Yao K(2015) A no-arbitrage theorem for uncertain stock model. Fuzzy optimization and decision making 14: 227-242.

Yao K(2021) Uncertain renewal process, 2th edition, Uncertainty theory laboratory.

Yu X(2012) A stock model with jumps for uncertain markets. International journal of uncertainty fuzziness and knowledge-based systems 20: 421-432. 